\title{
Artificial Semiconductor Superlattices and Quantum Well Structures
}

\author{
Klaus Ploog, Stuttgart
}

(Max-Planck-Institut für Festkörperforschung)

The recent developments in the thinfilm growth techniques of Molecular Beam Epitaxy (MBE) and Metalorganic Vapour Phase Epitaxy (MOVPE) make possible the synthesis of highly refined ultrathin multilayer structures composed of a periodic array of equally spaced hetero- or homojunctions in crystalline semiconductors (Fig. 1). The constituent layer thicknesses $L_{z}$ and $L_{b}$ have now been scaled down to atomic monolayers. In these artificial superlattices or multi-quantum well (MOW) structures, novel physical phenomena occur arising from quantum size effects. The energies and wavefunctions of electrons and holes are significantly modified as compared to bulk material, and quantized energy levels, called sub- or minibands, are formed. The free carriers are essentially confined in the plane of the layers and thus become two-dimensional. Consequently, the density-ofstates exhibits a staircase shape with step increases at the sub-band edges.

In Fig. 1 we show the periodic layer sequence of a $\mathrm{GaAs} / \mathrm{Al}_{\mathrm{x}} \mathrm{Ga}_{1-\mathrm{x}}$ As superlattice and the periodic variation of the corresponding conduction and valence band edges. This prototype superlattice represents an ideal system for theoretical analysis and for experiments to de- monstrate the effect of energy quantization on the optical and transport properties. The admixture of Al to the directgap semiconductor widens the energy gap of $\mathrm{Al}_{\mathrm{x}} \mathrm{Ga}_{1-x} \mathrm{As}$, but it produces only minor additional distortion because of the closely related chemical nature of $\mathrm{Al}$ and $\mathrm{Ga}$ atoms. This widening raises the conduction band edge and lowers the valence band edge, and thus potential barriers for both electrons and holes are created. The band gap discontinuity between $\mathrm{GaAs}$ and $\mathrm{Al}_{x} \mathrm{Ga}_{1-x} \mathrm{As}$ occurs mainly in the conduction band. To a good approximation the sub-band structure of this materials system can be obtained from simple calculations by wavefunction matching, using the effective masses $m^{*}$ of the Kronig-Penney model. The quantized energy is $h^{2} n^{2} / 8 m^{*} L_{z}^{2}$ for the $n$th confined state which contains $n$ electron half-wavelengths in a well thickness $L_{z}$. This value corresponds to the ideal case of infinitely high barriers, but is less for finite barrier heights. For a more accurate calculation the allowed electron and hole states can be determined by solving Schrödinger's equation and matching the wavefunctions to the appropriate boundary conditions.

The existence of quantized states or sub-bands in $\mathrm{GaAs} / \mathrm{Al}_{\mathrm{x}} \mathrm{Ga}_{1-\mathrm{x}}$ As multi-

Fig. 1 - Transmission electron micrograph of a (110) cleaved and thinned GaAs/AIAs superlattice of layer thicknesses $L_{2}=L_{b}=10 \mathrm{~nm}$.

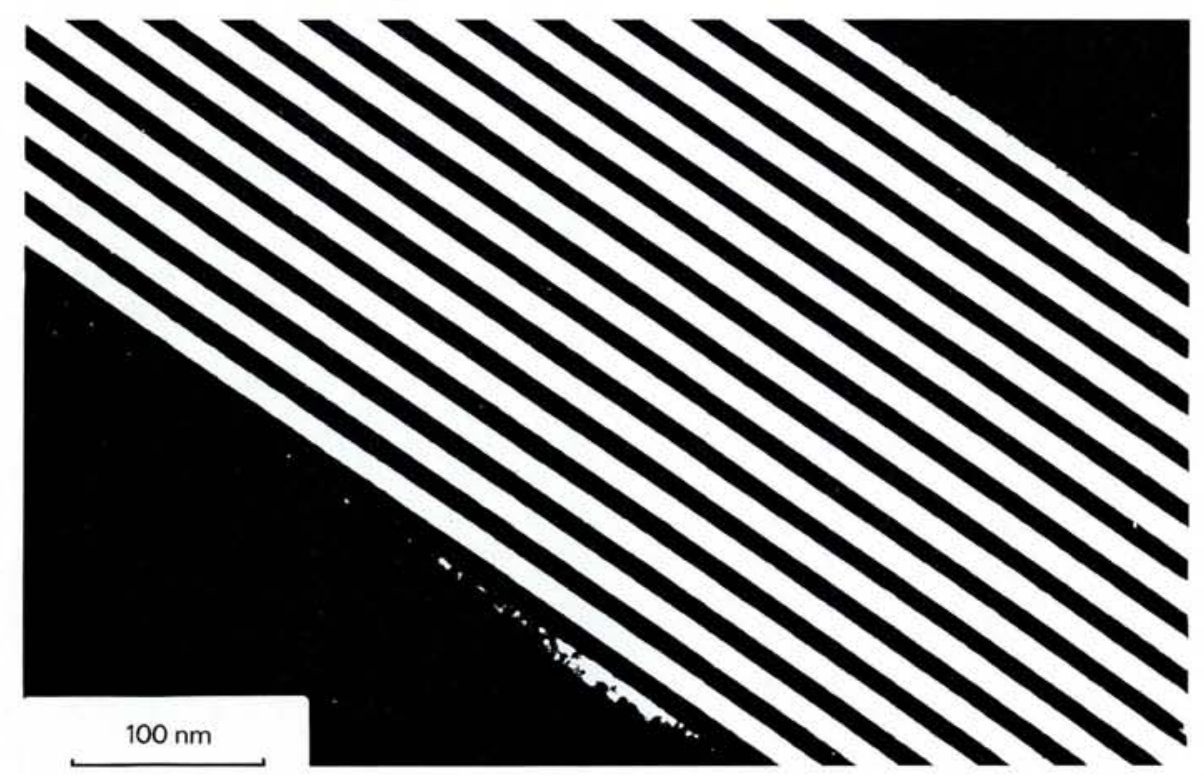

a)
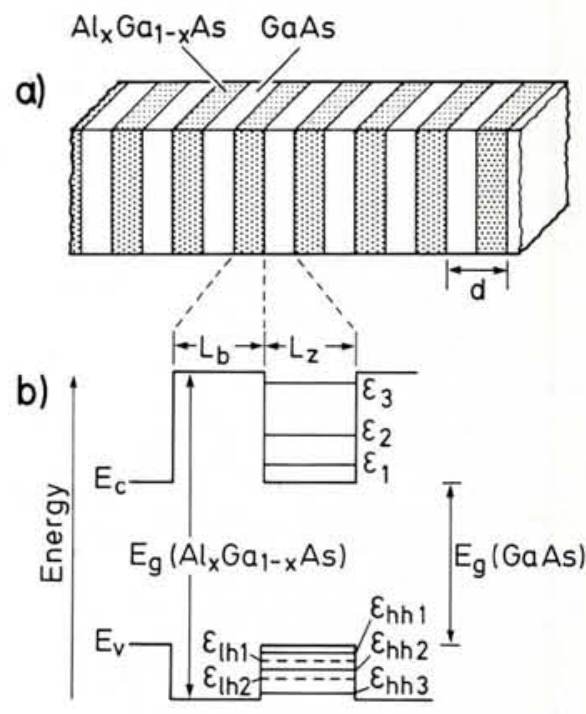

$\rightarrow \mathrm{z}$

Fig. 2 - (a) Periodic layer sequence of a $\mathrm{GaAs} / \mathrm{Al} \mathrm{Ga}_{\mathrm{x} . \mathrm{x}}$ As superlattice and (b) variation of the conduction and valence band edges. $\varepsilon_{i}, \varepsilon_{h h i}$ and $\varepsilon_{i h i}$ denote the subband energies.

quantum well structures can be directly probed by optical absorption measurements (Fig. 3). A series of absorption peaks is observed in samples of different configuration, owing to transitions from conduction sub-bands to valence subbands. The energy levels are shifted from the band gap to higher energy because of size quantization in the GaAs layers. The absorption spectra show contributions from both heavy and light holes, and also confirm the selection rules of the principal quantum number $\Delta n=0$ for such transitions. The data of Fig. 3 can be explained by the approximation that the electrons and holes are confined by square-well potentials arising from the heterojunction band discontinuities. This simple particle-in-thebox model accounts for the observed absorption spectra, if we assume that about $85 \%$ of the band-gap discontinuity occurs in the conduction band and about $15 \%$ in the valence band. It is further important to note that the binding energy of an exciton - a bound electron-hole pair with energy levels in the band gap which moves through the crystal lattice as a unit - is strongly enhanced. This may be factor of four for a 5-nm well, as compared to bulk GaAs, because of the two-dimensional confinement of electrons and holes. As a result, the excitonic features in the absorption spectra remain stable up to temperatures as high as $300^{\circ} \mathrm{C}$.

The $\mathrm{GaAs} / \mathrm{Al}_{\mathrm{x}} \mathrm{Ga}_{1-\mathrm{x}} \mathrm{As}$ superlattices and multi-quantum well structures are very attractive for solid state current excited lasers because light emission based on confined-particle transitions 
can be tailored to some energy between the band-gap of the GaAs well and that of the $\mathrm{Al}_{\mathrm{x}} \mathrm{Ga}_{1-\mathrm{x}} \mathrm{As}$ barrier material, simply by pre-selecting the well thickness and the alloy composition of the barrier during epitaxial growth. While in GaAs bulk material the dominant luminescence arises from electron-to-impurity recombination, the $\mathrm{GaAs}$ quantum well luminescence is dominated by intrinsic free-exciton recombination. Moreover, in quantum wells, the electron-hole recombination lifetime is reduced significantly, owing to carrier localization, so that the luminescence efficiency increases strongly. In Fig. 4 we show that the high-energy shift of the intrinsic freeexciton luminescence is proportional to the inverse square of the well width $L_{z}$. In addition to the blue shift we observe an increasing line width. This is caused by the constant thickness fluctuation within the GaAs quantum well of the order of one monolayer which has a stronger impact for narrower wells. The emission wavelength of $\mathrm{GaAs}$ quantum well lasers can easily be reduced by selection of a narrow well width. It is further important to note that in quantum well lasers the density-of-states is modified from the parabolic bulk-like distribution to a step-like distribution with constant density-of-states above the band edge up to the next excited sub-band. This modification of the density-of-states implies that significantly less carrier injection is required to reach threshold for stimulated emission, because in quantum wells more electron states are concentrated at energies near the band edge. Consequently, extremely low current threshold densities are observed for quantum well lasers. These new lasers also hold promise with respect to lasing lifetime.

In selectively doped $\mathrm{GaAs} / \mathrm{Al}_{\mathrm{x}} \mathrm{Ga}_{1-x} \mathrm{As}$ superlattices and quantum well structures, only the wider-gap semiconductor $\mathrm{Al}_{\mathrm{x}} \mathrm{Ga}_{1-\mathrm{x}} \mathrm{As}$ is doped with either $\mathrm{Si}$ donors or $\mathrm{Be}$ acceptors, while the smaller gap GaAs is left nominally undoped. Because of the conduction and valence band discontinuity at the heterointerface, the free carriers from the ionized dopant impurities in the $A I_{x} \mathrm{Ga}_{1-x} \mathrm{As}$ barrier are transferred to the adjacent undoped $\mathrm{GaAs}$ region where they form a quasi two-dimensional carrier gas. The spatial distribution of the dopant atoms and of the electrons as well as the relative energy positions of the band edges, donor levels and electron levels for a selectively $\mathrm{n}(\mathrm{Si})$-doped multi-quantum well structure is shown schematically in Fig. 5. In this configuration the electrons, which occupy quantum well states up to the Fermi level $E_{\mathrm{F}}$

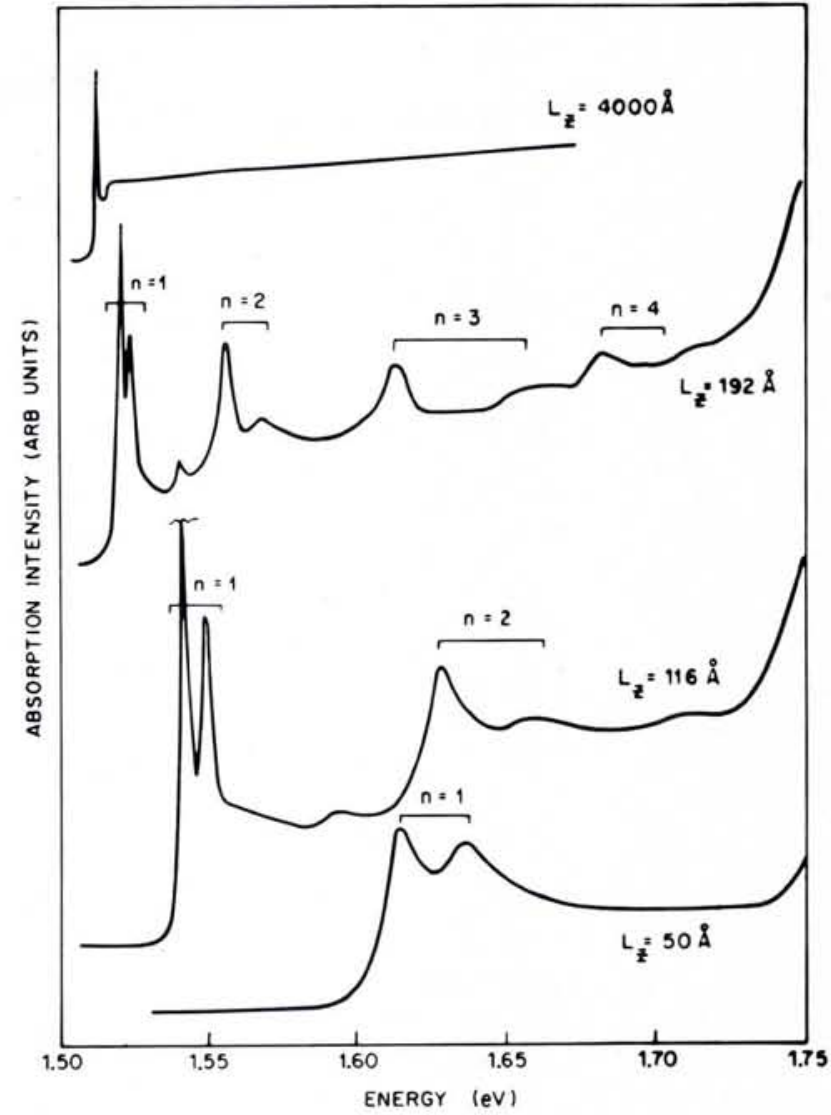

Fig. 3-Absorption spectra of

GaAs/Al Ga $_{1 \times \times}$ As multi quantum well struc. tures with different well widths $L_{z}$. The sample with $L_{2}=400$ $\mathrm{nm}$ shows bulk-type behaviour.

are spatially separated from their parent ionized dopant impurities. The electron mobilities in selectively doped $\mathrm{GaAs}$ / $A I_{x} \mathrm{Ga}_{1-x}$ As quantum well structures are greatly enhanced as compared to uniformly doped GaAs, particularly at low temperatures (Fig. 6). The reason for the increased mobilities is the reduction of scattering from ionized dopant impurities in the conducting channel, which is the dominant scattering mechanism at low temperatures. The ionized impurity scattering is further reduced if a thin ( 5 to $25 \mathrm{~nm}$ ) undoped spacer layer is inserted in the $\mathrm{Al}_{x} \mathrm{Ga}_{1-\mathrm{x}} \mathrm{As}$ barrier next to the conducting channel. In selectively $\mathrm{n}(\mathrm{Si})$-doped $\mathrm{GaAs} / \mathrm{Al}_{\mathrm{x}} \mathrm{Ga}_{1-\mathrm{x}} \mathrm{As}$ heterostructures with a single interface, lowtemperature electron mobilities in excess of $2 \times 10^{6} \mathrm{~cm}^{2} / \mathrm{V} \mathrm{s}$ have been achieved. In the complementary selectively $\mathrm{p}(\mathrm{Be})$-doped heterostructures the low-temperature hole mobilities are in the order of $10^{5} \mathrm{~cm}^{2} / \mathrm{V} \mathrm{s}$ owing to the larger effective hole mass. These figures are more than an order of magnitude higher than would be found in high purity bulk $n$ - and p-type GaAs.

Selective or modulation doping has enabled physicists to employ GaAs/ $\mathrm{Al}_{\mathrm{x}} \mathrm{Ga}_{1-\mathrm{x}} \mathrm{As}$ quantum well structures and heterostructures to study totally new fundamental problems, including the quantized Hall effect and the ordering of the two-dimensional electrons (or holes) in a new state, an incompressible electron (or hole) liquid. The excitations of this new state are apparently fractionally charged quasi-electrons and quasiholes. In addition, a new device concept, called High Electron Mobility Transistor (HEMT) has been developed from these selectively doped heterostructures for high-speed logic application.

Fig. 4 - Photoluminescence spectra of $\mathrm{GaAs} / \mathrm{Al}_{\mathrm{x}} \mathrm{Ga}$ 1.x As quantum well structures with different well widths $L_{z}$.

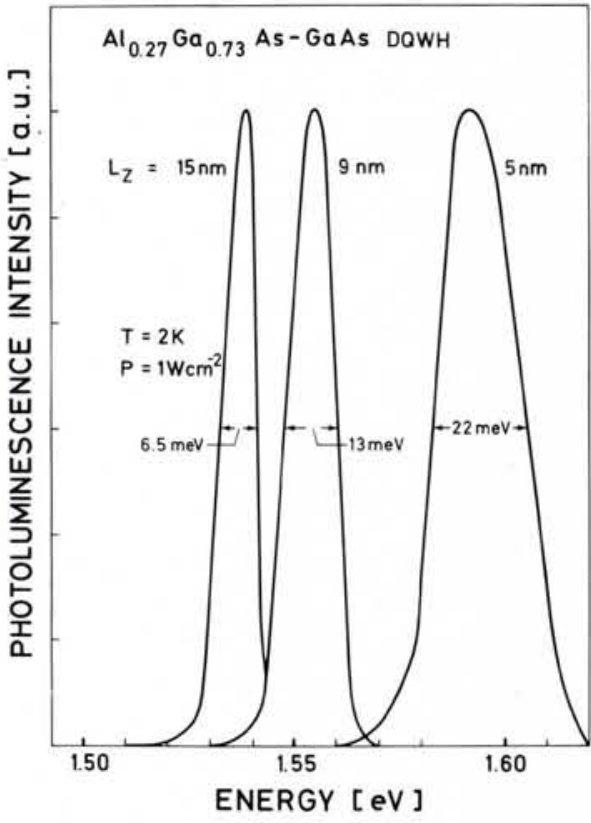




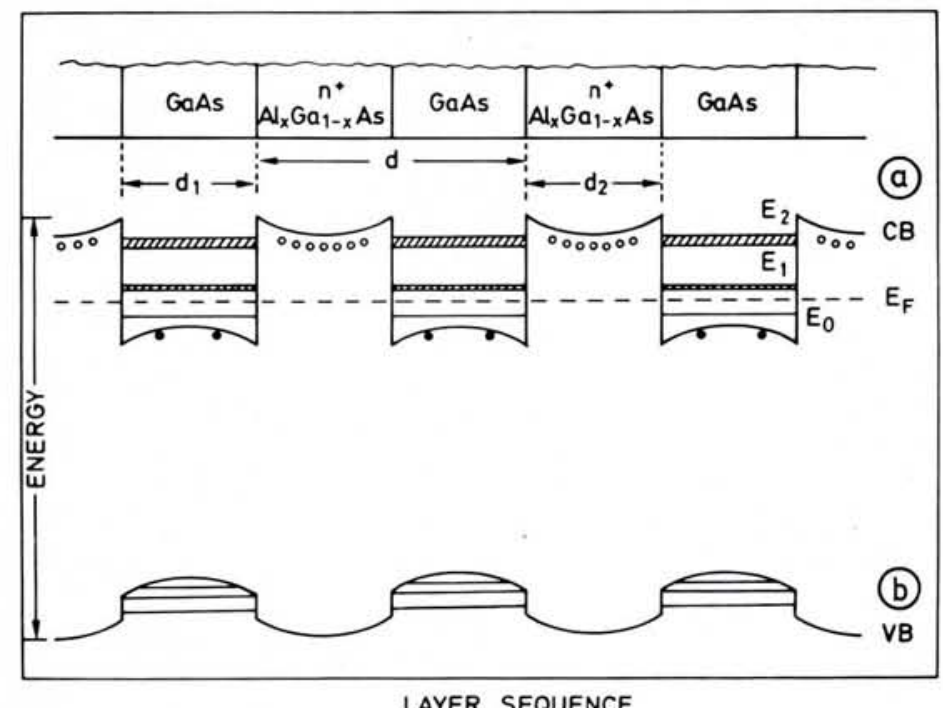

LAYER SEQUENCE

Fig. 5 - (a) Periodic layer sequence of a selectively $n(S i)$-doped GaAs/Al $\mathrm{Ga}_{1}$. As multi quantum well structure and (b) relative energy positions of the band edges, donor levels and electron levels.

At low magnetic fields the Hall effect is routinely used to measure carrier concentrations and mobilities in epitaxial semiconductor layers. However, if at low temperatures high magnetic fields are applied perpendicular to the two-dimensional electron or hole gas in selectively doped $\mathrm{GaAs} / \mathrm{Al}_{x} \mathrm{Ga}_{1-x}$ As structures, intriguing new features develop in the Hall response and conductivity. As indicated in Fig. 7, wide flat plateaux in Hall resistance and wide regions of vanishing resistance parallel to the current flow occur under variation of the perpendicular magnetic field. At each of the plateaux, the Hall resistance (i.e. Hall voltage divid-

Fig. 6 - Variation of Hall electron mobility with temperature in a selectively $n(S i)$ doped $\mathrm{GaAs} / \mathrm{Al}_{x} \mathrm{Ga}_{1-x}$ As multi quantum well structure.

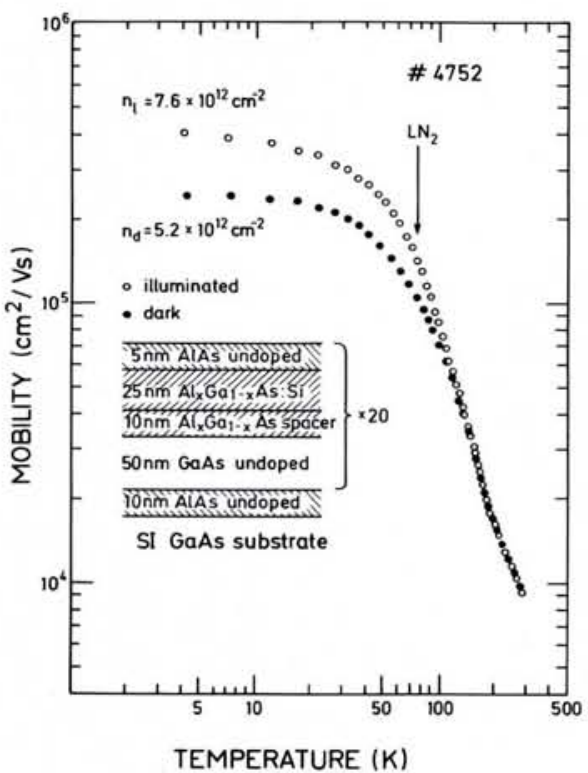

ed by current) is quantized in sub-multiples of $h / e^{2}$, with $e=$ electron charge and $h=$ Planck's constant. It is important to note that over a range of more than $1 \mathrm{kG}$ of applied magnetic field, the Hall resistance is constant and reproducible to within one part in $10^{8}$, independent of the exact geometry of the device. This accuracy of quantization makes the selectively doped Hall structures attractive for a new resistance standard and for determination of the fine structure constant $\alpha$, which is directly proportional to $e^{2} / h$ (Europhys. News 16 (1985) 11/12). A behaviour similar to that shown in Fig. 7 is observed in two-dimensional hole systems.

The physical reason for the peculiarity of a two-dimensional electron or hole gas in a strong magnetic field is that the energy spectrum of the electrons (holes) is discrete (formation of Landau levels) because of orbital quantization of the cyclotron motion in a magnetic field. Quantization of the Hall resistance to exact multiples of $h / e^{2}$ are observed at integer values of Landau level filling. Today, the integral quantum Hall effect is interpreted in terms of gaps in the singleparticle density-of-states between Landau levels. The formation of localized states in the gap region between magnetic levels owing to disorder (i.e. random distribution of impurities or interface steps) is assumed to account for the width of the plateaux and the width of the zero-resistance region in Fig. 7 .

At very low temperatures $(<1 \mathrm{~K})$ and high magnetic fields up to $30 \mathrm{~T}$ in the extreme quantum limit, where only the lowest Landau level contains carriers, striking new features are observed in high-mobility two-dimensional electron (hole) gas systems of selectively doped $\mathrm{GaAs} / \mathrm{Al}_{\mathrm{x}} \mathrm{Ga}_{1-x} \mathrm{As}$ structures: Quantization of the Hall resistance to exact rational fractions of $h / e^{2}$ occurs at fractional filling of the lowest Landau level. This observation indicates the formation of a new many-particle ground state, an electron (hole) liquid, at these fractional filling factors. Recent theoretical calculations suggest that this novel electronic state should have unusual properties like fractionally charged quasi-particles and resistance-less conduction at $T=0 \mathrm{~K}$. This fractional quantum Hall effect represents the first observation of a fractional quantum number in physics.

In many industrial laboratories extensive effort is now directed towards the development of High Electron Mobility Transistors (HEMT) and integrated circuits made from selectively doped $\mathrm{GaAs} / \mathrm{Al}_{\mathrm{x}} \mathrm{Ga}_{1-\mathrm{x}} \mathrm{As}$ quantum well structures. Although the entire low-field mobility enhancement is not available in devices operating at high electric fields under short gates, the HEMT has shown substantially higher speed than uniformly doped $\mathrm{GaAs}$ field effect transistors. In Fig. 8 we show the schematic arrangement of an experimental inverter structure with enhancement-mode switching HEMT and depletion-mode load HEMT, which was first realized by Fujitsu Ltd. (Japan). Ring oscillator circuits fabricated with such elements have achieved propagation delays down to $12 \mathrm{ps}$ at room temperature and $10 \mathrm{ps}$ at liquid nitrogen temperature $(77 \mathrm{~K})$. These are the highest speeds reported to date for a digital circuit. Recently, more realistic high-speed logic circuits based on the two-dimensional quantum confined electron channel in selectively doped

Fig. 7 - Variation of Hall resistance and parallel resistance with magnetic field in a selectively $n\left(\right.$ Si)-doped $G a A s / A I_{x} G a_{1-x} A s$ quantum well structure.

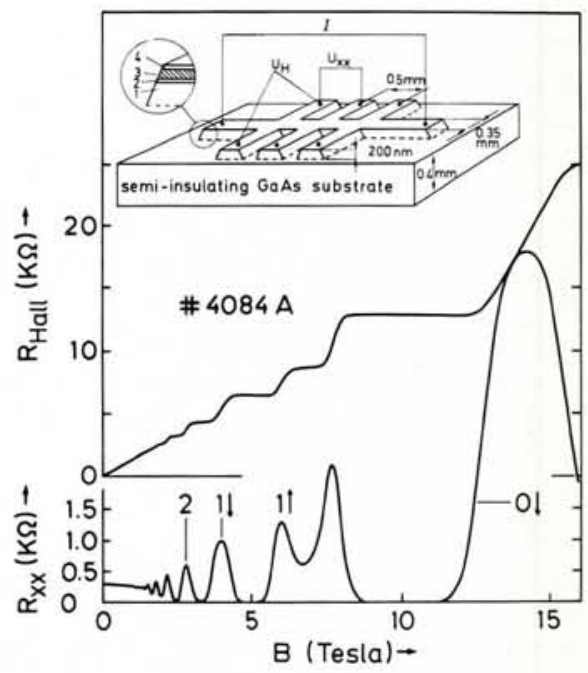


$\mathrm{GaAs} / \mathrm{Al}_{\mathrm{x}} \mathrm{Ga}_{1-\mathrm{x}} \mathrm{As}$ structures have been developed, including flip-flop frequency dividers with a dividing frequency of 5.5 $\mathrm{GHz}$ at room temperature and $10.1 \mathrm{GHz}$ at liquid-nitrogen temperature as well as a $4 \mathrm{~kb}$ HEMT SRAM ("Static Random Access Memory") with an address access time of $2.0 \mathrm{~ns}$ at liquid-nitrogen temperature.
Fig. 8-Schematic layer arrangement of experimental inverter structure based on High Electron Mobility Transistor (HEMT).

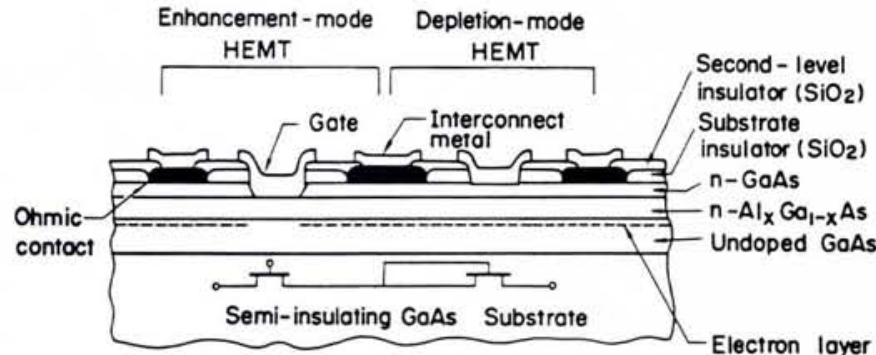

\section{New Individual Ordinary Members of EPS}

\section{CATEGORY 4a)}

P.G. Carolan, Abingdon, UK

A.J.H.A.E. Daneels, CERN, Geneva, $\mathrm{CH}$

\section{CATEGORY 4c)}

Austrian Physical Society

S. Kuhn, Innsbruck

Ch.J. Leubner, Innsbruck

M. Musso, Graz

\section{Belgian Physical Society}

J.-M. André, Malonne

F. Baras, Brussels

R. Dekeyser, Bertem

D.W. Faulconer, Brussels

P. Grobet, Heverlee

J.P. Lanquart, Brussels

F. Peeters, Antwerp

W. Rasker, Deurne

L. Renders, Hasselt

J. Steyaert, Louvain-la-Neuve

L. Vanneste, Leuven

G. Van Hooydonk, Gent

G. Van Wassenhove, Brussels

Eötvös Lorand Physical Society

C. Hajdu, Budapest

E. Jaroli, Budapest

E. Kotai, Budapest

T. Lohner, Budapest

\section{Finnish Physical Society}

A. Ahtee, Helsinki

B. Friman, Abo

V. Halonen, Oulu

K. Huitu, Helsinki

S. Islander, Helsinki

M. Kalliomäki, Helsinki

M. Kurkijärvi, Espoo

L. Lantto, Oulu

R. Orava, Helsinki

J. Raatikainen, Lappeenranta

H. Rajainmäki, Jyväskylä

C. Schultz, Espoo

L. Sonström, Emsalö

O. Syvähuoko, Helsinki

French Physical Society

R. Almairac, Montpellier
J. Bon, Lyon

R. Bougault, Caen

G. Coignet, Annecy-Le-Vieux

P. Damay, Lille

J. Desesquelles,

Rillieux-La-Pape

J. Druaux, Igny

J. Dumas, Lyon

A. Gerschel, Orsay

D. Gogny, Bruyères-Le-Chatel

G. Graner, Boulogne

H. Hommel, Paris

J.-C. Keller, Bures-sur-Yvette

R. Lallement, Paris

G. Laurence, St. Maur

A. Le Floch, Rennes

P. Louvet, Palaiseau

B. Malraison, Grenoble

G. Martin, Vitry

G. Perny, Strasbourg

J.-P. Petitet, Gagny

G. Pouyet, Paris

F. Rahimi, Mashad, Iran

M. Rey-Campagnolle,

Ferney-Voltaire

A. Salin, Talence

A. Schuhl, Antony

D. Sebilleau, Creteil

P. Simon, Orleans

$\mathrm{H}$. Theveneau, Boulogne

M. Tirelli, Palaiseau

P. Tronc, Paris

M. Veyssie, Paris

German Physical Society

W. Banzhaf, Stuttgart

H. Bruhns, Neckargemünd

H. Ehrhardt, Kaiserslautern

D.M. Herlach, Köln

J. Lühr, Hamburg

A. Otto, Düsseldorf

T. Schmickler, Munich

F. Steiner, Geneva, $\mathrm{CH}$

G. Weiss, Heidelberg

The Institute of Physics

R.D. Diehl, Liverpool

J. Higinbotham, Edinburgh

P.I.P. Kalmus, London

I.C. Malcolm, Edinburgh

D. Melville, Preston

P. Weightman, Liverpool
Israel Physical Societ

J. Goldberg, Haifa

Netherlands' Physical Society

$\mathrm{H}$. van Kempen, Nijmegen

L. Pietronero, Groningen

W. van Toledo, Nieuwegein

Italian Physical Society

R. Bonifacio, Milan

C. Brazzarotto, Varzo

G.H. Duffey, Brookings, SD, USA

F. Fedeli, Caltanissetta

M. Macri, Genoa

R. Pucci, Catania

N.A. Salingaros,

San Antonio, TX, USA

G. Salvini, Rome

M. Sampoli, Venice

Norwegian Physical Society

J. Feder, Oslo

Physical Section of the Yugoslav Association of Mathematicians, Physicists and Astronomers

B. Cabric, Kragujevac

Polish Physical Society

M. Lapucha, Warsaw

K. Lukierska-Walasek, Szczecin

K. Meissner, Warsaw

E. Soczkiewicz, Gliwice

W. Wislicki, Warsaw

Portuguese Physical Society

A. Almeida, Porto

Romanian National Committee for Physics

M. Crisan, Cluj

D. Poenaru, Bucharest

Spanish Royal Society of

Physics

M.T. Bariuso, Santander
A. Macias, Madrid

Swedish Physical Society

G. Grimvall, Stockholm

A. Holst, Lund

B. Höistad, Uppsala

F. Lettenström, Uppsala

N. Martensson, Uppsala

Swiss Physical Society

W. Buehrer, Endingen

$\mathrm{Ph}$. Choquard, Lausanne

C. Flesia-San Germano, Begnins

H.-J. Güntherodt, Basel

K. Knop, Zurich

M. Mayor, Sauverny

J.-H. Weisen, Lausanne

Turkish Physical Society

A.K. Atakan, Istanbul

\section{CATEGORY 4d)}

American Physical Society M.W. Cole, University Park, PA

P. Gagliardi, Prade, I

B. Hafizi, Boulder, $\mathrm{CO}$

E.U. Khan, Dera Ismail Khan,

Pakistan

G. Leppelmeier, Karslruhe, D

I.H. Mallender, Manchester, UK

M. Migliuolo, Rochester, NY

E. Oktay, Columbia, MD

D.E. Post, Princeton, NJ

J. Rafelski, Rondebosch,

Cape, SA

T.G. Rizzo, Ames, IA

M.L. Sawley, Lausanne, $\mathrm{CH}$

J.P. Schiffer, Argonne, IL

P. Schlein, Geneva, $\mathrm{CH}$

French Optical Society

P. Bouchareine, Paris

J. Bulabois, Besancon

P. Kayoun, Orsay

Physical Society of Japan

S. Nagao, Nagoya

M. Nishioka, Yamaguchi

T. Ikemura, Nara-Ken 